\title{
Infecção pelo estafilococo coagulase-negativa em recém-nascidos: mito ou verdade?
}

\author{
Coagulase-negative staphylococcus infection in newborns: myth or reality?
}

\author{
Ernani Miura*
}

C Pediatria um importante trabalho sobre significado clíniPediatria um importante trabalho sobre o significado clínico do estafilococo coagulase-negativa (ECN) em recémnascidos (RNs). Até duas décadas atrás, esta bactéria era considerada um comensal, no entanto, tornou-se patogênica na medida em que se universalizou o uso de antissépticos para a pele e coto umbilical, visando à redução da colonização por S. aureus; aumentou-se a sobrevida e o tempo de hospitalização de RNs prematuros, e o excessivo uso de meticilina (oxacilina) pressionou a produção de ß-lactamase e múltipla resistência aos antibióticos ${ }^{1}$.

D‘Angio et al. descreveram uma taxa de colonização por ECN entre $50 \%$ a $80 \%$ dos casos até 4 dias após a admissão dos RNs em unidade de tratamento intensivo neonatal (UTIN), e observaram que havia um aumento da resistência a múltiplos antibióticos de $32 \%$ para $82 \%$ no final de uma semana na $\mathrm{UTIN}^{2}$. Não há dúvidas de que o isolamento de ECN de amostras do sangue, liquor e urina de um RN com sinais e sintomas de sepse é significativo, porém, com muita freqüência pode representar uma contaminação no momento da coleta. Por isso, os autores recomendam para seu diagnóstico o isolamento do ECN em duas amostras de sangue, com resistência a mais de 6 antibióticos, incluindo a meticilina; ou ainda, identificando o mesmo ECN em duas amostras, coletadas simultaneamente de dois locais diferentes. Além disso, o ECN que cresce após 48 horas de incubação é freqüentemente um caso de contaminação ${ }^{3}$.

Entre 13 espécies encontradas no ser humano, o Staphylococcus epidermidis é clinicamente o mais importante para o RN. Essa bactéria, nos EUA, é responsável por cerca de $10 \%$ a $27 \%$ de todos os casos de sepse nas UTINs, com taxas de $55 \%$ em recém-nascidos de muito baixo peso

\footnotetext{
* Professor Adjunto-Doutor de Pediatria da FAMED-UFRGS.
}

$(\text { RNMBP } \leq 1.500 \text { gramas })^{1}$. As principais manifestações num estudo da sepse em prematuros foram apnéia e bradicardia ( $88 \%)$, necessidade de oxigênio (59\%) e ventilação mecânica (69\%), e os marcadores laboratoriais de fase aguda foram pouco sensíveis ${ }^{4}$. O quadro clínico inclui sepse, meningite com ou sem alterações no liquor, enterocolite necrosante, pneumonia, onfalite, abscesso de tecido mole, endocardite, e abscesso e osteomielite nos locais de venopunção. A mortalidade é baixa, concordante com os dados de Cunha et al., que descreveram uma mortalidade de $13 \%{ }^{1}$.

Os fatores de risco para a infecção por ECN incluem a presença de corpo estranho, referidos no artigo de Cunha como cateter venoso central, cateter umbilical, derivação ventrículo-peritonial, tubo endotraqueal, ventilação mecânica, dreno torácico, cateter de diálise peritonial, antibióticos prévios, sonda nasogástrica e nutrição parenteral, com inclusão de lipí$\operatorname{dios}^{1}$. A fisiopatogenia é devida à aderência que se estabelece entre a bactéria e o biopolímero do cateter, com a formação de um biofilme e uma produção copiosa de um muco viscoso de exopolissacarídeo. Este muco viscoso cobre a bactéria e a protege contra o antibiótico, imunoglobulinas e a opsoninofagocitose. A produção deste muco viscoso é significativamente maior na criança infectada, e pode aumentar se a dose do antibiótico (vancomicina e teicoplanina) for subinibitório ${ }^{5}$. O ECN produz uma variedade de exotoxinas que podem servir como fatores de virulência, incluindo hemolisinas, proteases, urease e fibrinolisina $^{6}$. Na enterocolite necrosante, identifica-se uma hemolisina semelhante à delta-toxina produzida pelo Staphylococccus aureus. Essa toxina produz uma lesão necrótica e sangramento na mucosa intestinal em ratos, e pode ser o responsável na patogênese da enterocolite do $\mathrm{RN}^{7}$.

No artigo de Cunha et al. foram isolados de 107 RNs, 117 vezes o $\mathrm{ECN}$, dos quais $51 \%$ foram considerados patogênicos e $49 \%$ contaminantes. Isso significou que na 
metade das vezes, o resultado não era verdadeiro. Entre os infectados, a maioria era prematuro (80\%), sendo a metade RNMBP, ou seja, com imaturidade imunológica. Além disso, estavam associados dois ou mais procedimentos invasivos, sendo que $89 \%$ tinham um cateter venoso central, $65 \%$ nutrição parenteral e $61 \%$ ventilação mecânica. A análise multivariada de regressão mostrou que um peso de nascimento $<1.500 \mathrm{~g}$ aumentava a chance de infecção em 6 vezes, a presença de corpo estranho aumentava a chance de infecção em 4,4 vezes, e o uso prévio de antibióticos aumentava a chance de infecção para 5,4 vezes mais. A espécie mais isolada foi o S. epidermidis em $78 \%$ dos casos, sendo presente em $87 \%$ das infecções e em $65 \%$ dos contaminantes. Silbert et al. relataram também, no Brasil, que entre $41 \mathrm{RNs}$ com hemocultura positiva para ECN, apenas $27 \%$ foram considerados infectados, sendo os demais $73 \%$ considerados como casos de contaminação ou casos duvidosos ${ }^{8}$. Portanto, a maior deficiência no diagnóstico de infecção por ECN é a contaminação no momento da coleta do material, sendo significativa para o sangue, e muito pior para corpo estranho e secreções, conforme se atesta nos trabalhos de Cunha e de Silbert.

$\mathrm{O}$ antibiótico de eleição no tratamento da sepse por ECN é a vancomicina, já que o ECN hospitalar é resistente à oxacilina e a cefalosporinas. Na presença de um cateter venoso central, é fundamental sua remoção, entretanto há situações em que não é possível sua retirada. Pode-se obter uma taxa de cura de até $80 \%$ com um antibiótico apropriado, sem a remoção do cateter ${ }^{9}$. O CDC-USA tem recomendado evitar o uso empírico da vancomicina, devido ao aumento de casos de enterococo e $S$. aureus resistente à vancomicina. Karlowicz et al. mostraram que, evitando o uso empírico de vancomicina, não houve diferenças nos resultados da sepse por ECN. No período I, de 1988 a 1994, foi usado vancomicina associada à cefotaxime, e no período II, de 1994 a 1997, foi usado oxacilina associada à gentamicina. Houve uma distribuição igual de casos de sepse fulminante ou óbito 1/141 (I) versus 1/136 (II). Os quatro principais agentes responsáveis pela morte por sepse foram Pseudomonas 20/36(50\%), E. coli 5/27 (19\%), Enterobacter $4 / 28$ (14\%) e Klebsiella 4/31 (13\%). Os autores concluem, recomendando focar o tratamento empírico para gramnegativo, particularmente Pseudomonas, e que a vancomicina somente deveria ser usada após a identificação do $\mathrm{ECN}^{10}$. Sinkowitz et al. verificaram que, entre 1993 a 1995, o serviço que mais utilizou a vancomicina foi o de Neonatologia, com uma taxa de uso de 34/100 admissões. Após, seguindo as recomendações do CDC e do Comitê de Controle de Infecção Hospitalar, houve uma redução do uso de vancomicina para 19/100 admissões $(p<0,001)$, sem alterar a morbimortalidade ${ }^{11}$.

Em casos de infecções graves, como a endocardite, ou ainda de má resposta por múltipla resistência do ECN, pode-se associar rifampicina à vancomicina, ou utilizar novos antibióticos, como novos glicopeptídeos (teicoplanina), a estreptogramina (quinuspristina/dalfopristina) ou ainda a oxazolidinonas (linezolida) ${ }^{1}$.
Assim, frente a uma infecção por ECN, se o paciente estiver em boa condição clínica, recomendamos manter a conduta adotada; se o paciente estiver piorando, trocar o antibiótico atual pela vancomicina, além de retirar os corpos estranhos e os lipídeos da nutrição parenteral e de reavaliar se houve quebras nas técnicas de prevenção de infecção hospitalar. Finalmente, as medidas mais importantes são as de prevenção - evitar o uso abusivo de antibióticos; lavagem obrigatória das mãos antes e após qualquer procedimento, reduzindo a transmissão desta bactéria entre o corpo clínico e o RN e entre os RNs hospitalizados; obedecer rigorosamente as técnicas cirúrgicas de redução de contaminação; atenção estrita aos protocolos de uso de cateteres venosos e arterial, e utilização de cateter percutâneo venoso central de pequeno calibre (PICC) para RNMBP, pois permite seu uso prolongado de 80 dias ou mais, com índice de infecção inferior a $10 \%{ }^{12}$.

\section{Referências bibliográficas}

1. Shinefield HR, St Geme III JW. Staphylococcal infections. In: Remington JS, Klein JO, editores. Infectious diseases of the fetus and newborn infant. $5^{\text {a }}$ ed. Philadelphia: WB Saunders Co.; 2001.p.1217-47.

2. D'Angio CT, McGowan KL, Baumgart S, St. Geme J, Harris MC. Surface colonization with coagulase-negative staphylococci in premature neonates. J Pediatr 1989;114:1029-35.

3. Awerwaldt LD, Geins M, Kao C, Pfaller MA. The positive predictive value of isolating coagulase-negative staphylococci from blood culture. Clin Infect Dis 1996;26:14-8.

4. Metzger-Maayan A, Linder N, Marom D, Visher T, Ashkenazi S, Sirota L. Clinical and laboratory impact of coagulase-negative staphylococcal bacteremia in preterm infants. Acta Paediatr 2000;89:690-3.

5. De Silva GD, Kantzanou M, Justice, Massey RC, Wilkinson AR, Day NP, et al. The ica operon and biofilm production in coagulasenegative Staphylococci associated with carriage and disease in a neonatal intensive care unit. J Clin Microbiol 2002;40:382-8.

6. Ishak MA, Groschel DHM, Mandell GL. Association of slime with pathogenicity of coagulase-negative staphylococci causing nosocomial septicemia. J Clin Microbiol 1985;22:1985-90.

7. Scheifele DW, Bjornson GL. Delta toxin activity in coagulasenegative staphylococci from the bowels of neonates. J Clin Microbiol 1988;26:279-84.

8. Silbert S, Rosa DD, Matte U, Goldim JR, Barcellos SH, Procianoy RS. Staphylococcus $s p$ coagulase-negativa em hemoculturas de pacientes com menos de sessenta dias de idade: infecção versus contaminação. J Pediatr (Rio J) 1997;73:161-5.

9. Prince A, Heller B, Levy J, Heird WC. Management of fever in patients with central venous catheters. Pediatr Infect Dis J 1986; 5:20-7.

10. Karlowicz MG, Buescher ES, Surka AE. Fulminant late-onset sepsis in a neonatal intensive care unit 1988-1997, and the impact of avoiding empiric vancomycin therapy. Pediatrics 2000; 106:1387-90.

11. Sinkowitz RL, Keyeserling H, Walker TJ, Holland J, Jarvis WR. Epidemiology of vancomycin usage at a children's hospital, 1993 through 1995. Pediatr Infect Dis J 1997; 16:485-9.

12. Durand M, Ramanathan R, Martinelli B, Tolentino M. Prospective evaluation of percutaneous central venous Silastic catheters in newborn infants with birth weight of 510 to 3,920 grams. Pediatrics 1986; 78:245-50. 